\title{
Development
}

\section{Dopaminergic Co-Regulation of Locomotor Development and Motor Neuron Synaptogenesis is Uncoupled by Hypoxia in Zebrafish}

\author{
Jong-Hyun Son, ${ }^{1}$ Tamara J. Stevenson, ${ }^{2}$ Miranda D. Bowles, ${ }^{2}$ Erika A. Scholl, ${ }^{2}$ and \\ (DJoshua L. Bonkowsky 2,3
}

https://doi.org/10.1523/ENEURO.0355-19.2020

${ }^{1}$ Department of Biology, University of Scranton, Scranton, PA 18510, ${ }^{2}$ Department of Pediatrics, University of Utah School of Medicine, Salt Lake City, UT 84132, and ${ }^{3}$ Brain and Spine Center, Primary Children's Hospital, Salt Lake City, UT 84108

\begin{abstract}
Hypoxic injury to the developing human brain is a complication of premature birth and is associated with longterm impairments of motor function. Disruptions of axon and synaptic connectivity have been linked to developmental hypoxia, but the fundamental mechanisms impacting motor function from altered connectivity are poorly understood. We investigated the effects of hypoxia on locomotor development in zebrafish. We found that developmental hypoxia resulted in decreased spontaneous swimming behavior in larva, and that this motor impairment persisted into adulthood. In evaluation of the diencephalic dopaminergic neurons, which regulate early development of locomotion and constitute an evolutionarily conserved component of the vertebrate dopaminergic system, hypoxia caused a decrease in the number of synapses from the descending dopaminergic diencephalospinal tract (DDT) to spinal cord motor neurons. Moreover, dopamine signaling from the DDT was coupled jointly to motor neuron synaptogenesis and to locomotor development. Together, these results demonstrate the developmental processes regulating early locomotor development and a requirement for dopaminergic projections and motor neuron synaptogenesis. Our findings suggest new insights for understanding the mechanisms leading to motor disability from hypoxic injury of prematurity.
\end{abstract}

Key words: axon pathfinding; hypoxia; prematurity; synaptogenesis

\section{Significance Statement}

Hypoxic injury in premature infants affects millions of infants and leads to long-term disabilities including problems with motor function. That hypoxia disrupts locomotor function has been known, but the mechanisms mediating hypoxia's effects have yet to be elucidated. Here we investigate the role of descending projections from the dopaminergic diencephalospinal tract (DDT), a group of highly conserved neurons, which in zebrafish are necessary for normal swimming behavior. We found that the DDT projects to spinal motor neurons, and that hypoxia leads to reduced swimming behavior and a concomitant decrease in DDT to motor neuron synapses. These results offer new insight into the evolutionarily conserved neuronal operators critical for locomotor development in vertebrates, and reveal a molecular mechanism of hypoxic injury.

\section{Introduction}

Development of the CNS depends on precise regulation of oxygen levels. Absence of oxygen (anoxia) or low

Received August 26, 2019; accepted January 17, 2020; First published January 30, 2020.

The authors declare no competing financial interests. oxygen levels (hypoxia) can cause devastating effects on development, and a concomitant range of molecular,

Author contributions: J.-H.S. and J.L.B. designed research; J.-H.S., T.J.S., M.D.B., and E.A.S. performed research; J.-H.S. and T.J.S. contributed unpublished reagents/analytic tools; J.-H.S., T.J.S., M.D.B., E.A.S., and J.L.B. analyzed data; J.-H.S., E.A.S., and J.L.B. wrote the paper. 
cellular, and neuronal changes (Bonkowsky and Son, 2018). Hypoxia is a major complication associated with premature birth, and can lead to permanent neurologic impairments including attention-deficit hyperactivity disorder, autism, cerebral palsy, motor impairment, epilepsy, or intellectual disability (Bass et al., 2004; Barrett et al., 2007; Saigal and Doyle, 2008; Back, 2014; Salmaso et al., 2014). Each year worldwide an estimated 15 million infants are born prematurely ( $<37$ weeks' gestation; Di Fiore et al., 2016). While survival rates for premature infants have drastically improved and the total number of ex-premature infants has increased over the past decade (Mathews et al., 2011), therapies to treat neurodevelopmental diseases resulting from prematurity have been lacking (Fanaroff et al., 2007; Hintz et al., 2011).

Accumulating evidence has shown that hypoxia causes altered neuronal connectivity in the brains of children born prematurely (Gozzo et al., 2009; Mullen et al., 2011). However, the mechanisms linking altered neuronal connectivity, defined as axon pathfinding and synaptic connectivity, to changes in motor function or behavior in vertebrates are still poorly understand. Previous work has shown that developmental hypoxia causes axon pathfinding errors in commissural telencephalic neurons, due to activation of the hypoxia-inducible factor (hif1) pathway in the developing zebrafish brain (Stevenson et al., 2012; Xing et al., 2015). In vivo as well as in vitro studies have demonstrated that hypoxia affects synapse development as well (Curristin et al., 2002; Valdez et al., 2007; Milash et al., 2016; Segura et al., 2016; Son et al., 2016). What has been lacking, however, is an understanding of how changes in connectivity in vertebrates alter behavior. In contrast, experiments in the nematode Caenorhabditis elegans have shown that altered connectivity after hypoxia causes behavioral changes including altered responses to sensory stimuli (Chang and Bargmann, 2008; Pocock and Hobert, 2010).

The vertebrate motor system offers a well-characterized model for understanding functional effects of hypoxia-associated connectivity changes. Development of motor function is a tightly regulated process, involving genetically encoded programs and specification of neuronal types and connections (Garcia-Campmany et al., 2010), but also feedback from environmental pathways, such as central pattern generators (CPGs; Berg et al., 2018; D'Elia and Dasen, 2018). The neurotransmitter dopamine has been identified as a critical mediator in several distinct aspects of motor development: dopamine is a brain-derived

This work was supported by the National Institutes of Health Grant DP2 $\mathrm{MH100008.} \mathrm{J.L.B.} \mathrm{was} \mathrm{supported} \mathrm{by} \mathrm{the} \mathrm{Bray} \mathrm{Chair} \mathrm{in} \mathrm{Child} \mathrm{Neurology}$ Research.

Acknowledgements: We thank M. Hobbs and the Centralized Zebrafish Animal Resource for animal care and M. Redd and C. Rodesch for assistance with microscopy.

Correspondence should be addressed to Joshua L. Bonkowsky at joshua. bonkowsky@hsc.utah.edu or Jong-Hyun Son at jong-hyun.son@scranton.edu.

https://doi.org/10.1523/ENEURO.0355-19.2020 Copyright (C) 2020 Son et al.

This is an open-access article distributed under the terms of the Creative Commons Attribution 4.0 International license, which permits unrestricted use, distribution and reproduction in any medium provided that the original work is properly attributed. signaling factor affecting neurogenesis in the spine (Popolo et al., 2004; Reimer et al., 2013); descending projections from the dopaminergic diencephalospinal tract (DDT) are required for vertebrate locomotor maturation; and it is required for regulation of locomotion (Jay et al., 2015; Sharples et al., 2015). Further, tyrosine hydroxylase (TH) immunoreactivity, a marker for synthesis of dopamine, has been shown juxtaposed to motor neurons (McLean and Fetcho, 2004a).

To explore hypoxia's roles on the interrelationship of motor function and circuitry, we investigated changes in the DDT and motor neuron connectivity, and accompanying effects on locomotor development. We developed transgenic animals expressing fluorescently tagged markers in the DDT and motor neurons to probe for colocalization and proximity at synapses. We found that DDT synaptic proteins immediately juxtapose motor neuron synapses. Since previous studies had shown that hypoxia affected DDT synapses (Son et al., 2016), we characterized the effects of developmental hypoxia on the neurons and circuitry of the DDT and motor neurons. We found no change between hypoxic and normoxic conditions in the number of motor and dopaminergic neurons, or in the axon projections of the DDT to the spinal cord. However, in hypoxic conditions there was a decrease in the number of synapses observed between the DDT and motor neurons. The loss of motor neuron synapses corresponded to a decrease in swimming behaviors compared with normoxic conditions. The impairment in swimming behavior persisted into adulthood, suggesting that developmental hypoxic injury leads to permanent changes in circuitry controlling locomotion.

\section{Materials and Methods}

\section{Ethics statement}

All zebrafish experiments were performed in accordance with guidelines from our institute's. Animal Care and Use Committee, regulated under federal law (the Animal Welfare Act and Public Health Services Regulation Act) by the United States Department of Agriculture (USDA) and the Office of Laboratory Animal Welfare at the National Institutes of Health, and accredited by the Association for Assessment and Accreditation of Laboratory Animal Care (AAALAC) International.

\section{Fish stocks, animal husbandry, transgenic line generation}

Adult fish were bred according to standard methods. Embryos were raised at $28.5^{\circ} \mathrm{C}$ in $\mathrm{E} 3$ embryo medium with methylene blue, and embryos beyond $24 \mathrm{~h}$ postfertilization (hpf) were treated with phenylthiourea (PTU) to prevent pigment formation. For in situ staining and immunohistochemistry, embryos were fixed in $4 \%$ paraformaldehyde (PFA) in PBS overnight $(\mathrm{O} / \mathrm{N})$ at $4^{\circ} \mathrm{C}$, washed briefly in PBS with $0.1 \%$ Tween 20 , dehydrated stepwise in methanol (MeOH; 25\%, 50\%, 75\%, 100\%), and stored in $100 \% \mathrm{MeOH}$ at $-20^{\circ} \mathrm{C}$ until use.

Transgenic fish lines and alleles used in this paper were the following: Tg(otpb.A:Gal4-VP16 413 - 470; myl7:EGFP) ${ }^{2057}$ 
(referred to as Tg(optb.A:Ga/4); Stevenson et al., 2012); $\mathrm{Tg}$ (zcUAS:PSD95.FingR-GFP-ZFC(CCR5TC)-KRAB(A)) zc88 (Son et al., 2016); Tg(otpb.A:EGFP-caax) ${ }^{\mathrm{zc} 49}$ (Gutnick et al., 2011); and Tg(myl7:EGFP; UAS:TagRFP-caax $)^{\text {zc61 }}$ (Xing et al., 2015). The lines Tg(otpb.A:TRFP-caax) ${ }^{2 c 97}$ and $\operatorname{Tg}\left(H b 9: G a l 4 V P 16_{413-470}\right)^{z c 98}$ (referred to as $\operatorname{Tg}(H b 9$ : Gal4)) were generated for this project. Cloning for use in zebrafish was based on the Tol2 kit and recombination reactions with Gateway (Invitrogen) plasmids (Kwan et al., 2007). Identity of constructs was confirmed by restriction enzyme digests and by sequencing of both strands (for coding sequences). Injection of DNA constructs and generation of stable transgenic lines was performed using standard protocols (Bonkowsky et al., 2008). Lines are available on request from the Zebrafish International Resource Center (ZIRC, Eugene, OR) or from the authors.

\section{Hypoxia reagents}

For hypoxia experiments, we followed settings and conditions established in previously published protocols (Stevenson et al., 2012; Xing et al., 2015; Gao et al., 2018). In brief, embryonic zebrafish were placed in a sealed Plexiglas chamber connected via a controller that monitored and adjusted nitrogen gas flow to a desired $\mathrm{pO}_{2}$ set point (Biospherix Ltd.). We observed that equilibration of oxygen partial pressures in water could take several hours measured with a dissolved oxygen water meter (Control Company). Therefore, we pre-equilibrated all solutions to either normoxia or hypoxia for at least $4 \mathrm{~h}$ before use, and transferred embryos into and out of preequilibrated solutions. At the desired time, embryos were placed into media that had been equilibrated to the hypoxic conditions. To terminate hypoxia, embryos were returned to media kept in normoxic conditions. Morphologic staging was used to help determine age at fixation for analyses.

\section{Spontaneous swimming behavior analysis}

Larval behavior analysis was performed on $5 \mathrm{~d}$ postfertilization (dpf) larvae in 96-well square bottom plates (Krackeler Scientific) using a video analysis software program (Noldus EthoVision). All experiments were performed in temperature-controlled room dedicated for behavior analysis. For spontaneous behavior observation, animals were transferred at $5 \mathrm{dpf}$ to the 96-well plate for 30-min acclimations prior to recording of behavior. The spontaneous behavior was measured for a total of $70 \mathrm{~min}$, including under alternating dark $(4 \times 10$ min) and light $(3 \times 10 \mathrm{~min})$ conditions and a one-way ANOVA was conducted.

\section{Immunohistochemistry and double immunohistochemistry}

Antibodies used were: rabbit polyclonal anti-TH 1:250 (Millipore AB152), rabbit polyclonal Hb9 1:250 (ThermoFisher) chicken monoclonal anti-GFP 1:250 (Aveslab, GFP1020), rabbit polyclonal anti-phospho-histone H3 (Thr3; EMD Millipore), rabbit anti-TagRFP 1:250 (Evrogen, AB234), Cy-3 anti-rabbit 1:400 (Millipore, AP132C), Alexa Fluor
488 Goat anti-chicken 1:1000 (ThermoFisher, A11039). Double immunohistochemistry for GFP and TH was performed as follows: embryos were fixed in 4\% PFA in PBS for $1.5 \mathrm{~h}$ at room temperature (RT) and washed briefly in PBS $(3 \times 5 \mathrm{~min})$ with $0.1 \%$ Triton $\mathrm{X}-100$ (PBST). Embryos were then blocked (PBS with 1\% BSA, 1\% DMSO, $2 \%$ goat serum, and $0.1 \%$ Triton $\mathrm{X}-100$ ) for $3 \mathrm{~h}$ at RT, and then incubated in blocking buffer with primary antibodies overnight at $4^{\circ} \mathrm{C}$. Embryos were washed with PBST, and incubated with secondary antibodies overnight at $4^{\circ} \mathrm{C}$.

\section{Terminal deoxynucleotidyl transferase dUTP nick-end labeling (TUNEL) staining}

TUNEL was performed on whole-mount larvae (ApopTag Fluorescein In Situ Apoptosis Detection kit; Millipore Bioscience Research Reagents). After standard fixation and dehydration of larvae in $100 \% \mathrm{MeOH}$, larvae were rehydrated stepwise into PBS with $0.1 \%$ Tween 20 (PBST), permeabilized with $10 \mathrm{mg} / \mathrm{ml}$ Proteinase $\mathrm{K}$ in PBST at $28^{\circ} \mathrm{C}$, washed twice with PBST, refixed for 20 min with $4 \%$ PFA, and washed with PBST. Subsequently, $75 \mu$ l of equilibration buffer was added to the larvae for $1 \mathrm{~h}$ and then removed and replaced with $55 \mu \mathrm{l}$ of "working-strength" (per Apoptosis Detection kit instructions) terminal deoxynucleotidyl transferase enzyme overnight at $37^{\circ} \mathrm{C}$. To avoid drying out the larvae, Eppendorf tubes were sealed with Parafilm. Before use, the anti-digoxigenin conjugate was warmed to $\mathrm{RT}$. The end-labeling reaction was stopped by washing the embryos three times for $15 \mathrm{~min}$ each with $2 \mathrm{ml}$ of the stop/ wash buffer, followed by three 1-min washes with PBS. Then $65 \mathrm{ml}$ of working-strength sheep anti-digoxigenin rhodamine was added to the embryos overnight at $4^{\circ} \mathrm{C}$. Double immunohistochemistry for GFP and TUNEL was performed by TUNEL staining; after washes with PBST, larvae were fixed for $20 \mathrm{~min}$ in $4 \%$ PFA at RT and washed again with PBST.

\section{Western blot analysis}

Thirty larvae (5 dpf) per group were deyolked by triturating and incubating in deyolking buffer $(65 \mathrm{~mm} \mathrm{NaCl}$, $1.7 \mathrm{~mm} \mathrm{KCl}$, and $1.5 \mathrm{~mm} \mathrm{NaHCO}_{3}$ ) for $10 \mathrm{~min}$ at RT. Protein was extracted by grinding larval fish with pestle in a $100 \mu$ l of lysis buffer $(150 \mathrm{~mm} \mathrm{NaCl}, 20 \mathrm{~mm}$ Tris-HCl, pH 7.5, 1 mm EDTA, $1 \%$ NP-40, and 1\% Triton X-100, $1 \times$ Halt Protease and Phosphatase Inhibitor Cocktail; Life Technologies) on ice. The extract was centrifuged for $20 \min (16,000 \times g \mathrm{RCF})$ at $4^{\circ} \mathrm{C}$ and the supernatant was transferred to a new tube. Total protein concentration was determined by BCA assay (Thermo Scientific Pierce BCA Protein Assay kit, Fisher Scientific). The protein extract was mixed with an equal amount of $2 \times$ Laemmli buffer [20\% glycerol, 4\% SDS, $0.1 \%$ bromophenol blue, $0.125 \mathrm{M}$ Tris (pH 6.8), and $2.5 \% \beta$-mercaptoethanol] and boiled for $3 \mathrm{~min}$. Samples were stored at $-20^{\circ} \mathrm{C}$ until use. A total of $20 \mu \mathrm{g}$ of total protein was loaded on to each lane of a 4-20\% polyacrylamide gel (Mini-Protean TGX Gel Bio-Rad). After electrophoresis proteins were transferred to PVDF membranes and blocked in 3\% non- 
fat dry milk in TBS $(50 \mathrm{~mm}$ Tris, $0.138 \mathrm{M} \mathrm{NaCl}$, and $2.7 \mathrm{~mm} \mathrm{KCl}$; $\mathrm{pH} 8.0$ ) for $30 \mathrm{~min}$ at RT with agitation. Membranes were split, and then incubated in rabbit anti-TH (1:1000) or rabbit anti- $\beta$-actin (1:1000) for $2 \mathrm{~h}$ at RT, washed in TBS, and then incubated in HRP-antirabbit (1:5000 dilution) for $1 \mathrm{~h}$ at RT. Following incubation with secondary antibody, membranes were washed extensively in TBS containing $0.05 \%$ Tween 20 , and then subjected to chemiluminescent detection (Clarity Western ECL Substrate; Bio-Rad). The Western blot analysis, imaging, and quantification were performed with a digital imager (Gel Do XR+System and Image Lab Software, Bio-Rad) in three separate experimental replicates.

\section{Microscopy, image analysis, and movie analysis}

Confocal imaging was performed, and quantification was performed in Image $J$ by compiling a sum projection of 75 slices (step size $2.0 \mu \mathrm{m}$ ) into a single z-stack image. For counts at 72 hpf a $340 \times 170$-pixel box was drawn in the telencephalon at with the ventral edge placed on the dorsal edge of the eye and the rostral edge at the nose; at 30 and 48 hpf, a $450 \times 300$-pixel box was drawn around the entire brain. Thresholding was applied from a minimum of 125 to a maximum of 255; and the "Analyze Particles" function was used for quantification.

\section{Statistical analysis}

All data are presented as means \pm SEM. Analyses comparing dependent measures across hypoxia and normoxia were completed with Student's $t$ test and one-way ANOVA followed by post hoc Tukey test. Overall, statistical significance was set at $p \leq 0.05$.

\section{Results}

\section{Descending dopaminergic axons form synapses adjoining spinal cord motor neurons}

We examined the projections of DDT axons, and the proximity of their synapses to spinal cord motor neurons in wild-type zebrafish at $5 \mathrm{dpf}$. We observed TH-positive $\left(\mathrm{TH}^{+}\right)$immunostaining juxtaposed with anti-Hb9-positive $\left(\mathrm{Hb}^{+}\right)$staining of motor neurons in the spinal cord (Fig. $1 A)$. To test the source of the $\mathrm{TH}^{+}$immunoreactivity, we co-labeled axons in the Tg(otpb.A:EGFP-caax) line, which expresses GFP in the descending DDT (Fujimoto et al., 2011). We found co-expression of $\mathrm{TH}^{+}$and GFP in the DDT (Fig. 1B). Next, to evaluate for potential synapse formation between the DDT and motor neurons, we used double transgenic larvae in which a GFP-tagged intrabody (FingR; Gross et al., 2013; Son et al., 2016) labeled the endogenous postsynaptic density 95 (PSD-95) protein at the postsynaptic motor neuron, and RFP-caax labeled the DDT. We found co-localized punctate labeling in the spinal cord of Tg(otpb.A:TRFP-caax); $\operatorname{Tg}(\mathrm{Hb}$ 9:FingR (PSD95)-GFP) larvae, suggesting the presence of synapses between the DDT and motor neurons (Fig. 1C, arrowheads). Interestingly, we identified co-localization of the dopamine receptor 4 (D4R) with the punctate labeling of FingR (PSD95) on motor neurons (Fig. 1D). The D4R has been shown to mediate the developmental requirement for dopamine in locomotor maturation and in neurogenesis of motor neurons (Lambert et al., 2012; Reimer et al., 2013). Together, these data suggest the presence of synapses between the DDT and motor neurons in the developing zebrafish spinal cord.

\section{Hypoxia reduces swimming activity}

The DDT is required for locomotor development, and because of our observation of potential synapses between the DDT and motor neurons, we were interested in whether disruption of DDT connectivity would affect motor development. In particular, since hypoxia in developing zebrafish results in pathfinding errors (Stevenson et al., 2012; Xing et al., 2015), and hypoxia has been associated with long-term motor impairment in human infants born prematurely (Martin et al., 2011b; Salmaso et al., 2014), we were interested in whether hypoxia would affect DDT connectivity and lead to resultant swimming deficits. We exposed developing zebrafish larvae to different sublethal hypoxic conditions including $1 \% \mathrm{pO}_{2}$ from 1 to 2 dpf, $3 \% \mathrm{pO}_{2}$ from 2 to $3 \mathrm{dpf}$, and $5 \% \mathrm{pO}_{2}$ from 3 to $4 \mathrm{dpf}$. The timing and percentage hypoxia was based on previous work, in which these conditions were shown to be non-lethal, and to be non-disruptive to overall development including aspects of CNS development such as cell fate specification and progenitor specification (Stevenson et al., 2012; Xing et al., 2015). Following hypoxia, animals were returned to normoxia, and behavior was then examined at $5 \mathrm{dpf}$ (as the developmental switch in the episodic locomotor (swimming) pattern occurs around $5 \mathrm{dpf}$; Fig. 2A; Buss and Drapeau, 2001; Lambert et al., 2012). Spontaneous swimming behavior was measured for $70 \mathrm{~min}$, including under alternating dark $(4 \times 10 \mathrm{~min})$ and light $(3 \times 10 \mathrm{~min})$ conditions (Fig. $2 \mathrm{~B})$. A one-way ANOVA showed effects of hypoxia on motor behavior, including swimming distance in dark $\left(F_{(3,111)}=11.930, p<0.01\right)$ and in light $\left(F_{(3,111)}=14.069, p<0.01\right)$; rotation frequency in dark $\left(F_{(3,111)}=2.860, p<0.05\right)$ and in light $\left(F_{(3,111)}=6.989\right.$, $p<0.01)$; and velocity in dark $\left(F_{(3,111)}=12.175, p<0.01\right)$ and in light $\left(F_{(3,111)}=13.793, p<0.01\right)$. Post hoc comparison using the Tukey HSD test indicated that spontaneous swimming behavior of larval zebrafish exposed to hypoxia was significantly decreased compared with that of larval zebrafish exposed to normoxia $\left(21 \% \mathrm{pO}_{2}\right.$; Fig. $2 \mathrm{C}$; Table 1). Taken together, these results suggest that hypoxia decreases the spontaneous swimming behavior of larval zebrafish and impacts motor development.

Importantly, we also found that the motor impairments caused by hypoxia persisted into adulthood. In adult fish that had been exposed to hypoxia as 3- to 4-dpf larvae, we also found significant decreases in distance swam $\left(t_{(16)}=2.9, p<0.05\right)$ and velocity $\left(t_{(16)}=2.5, p<0.05\right.$; but not in total time spent swimming $\left(t_{(16)}=1.1, p=0.27\right.$; Fig. $2 D$; Table 2). Together, these results are consistent with hypoxia causing deficits in motor behavior that are sustained through development into adulthood. Further, this suggests that specific changes in motor circuitry may be responsible for the impairment in behavior. 
A

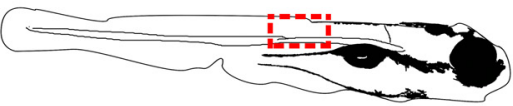

TL WT
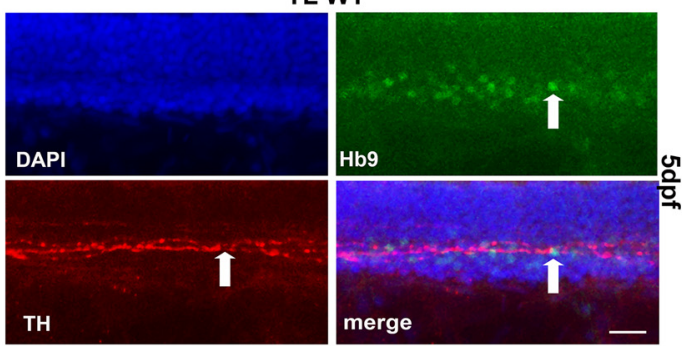

C
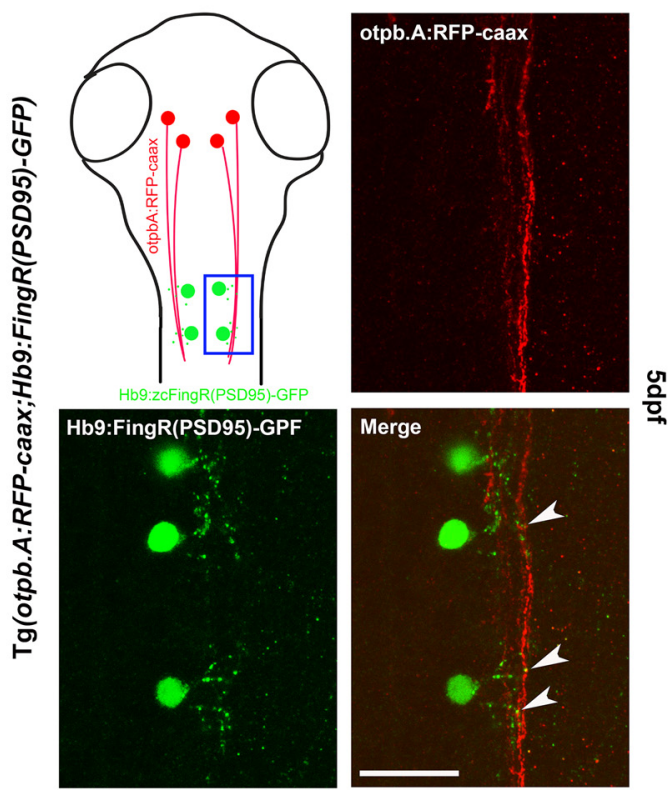

B
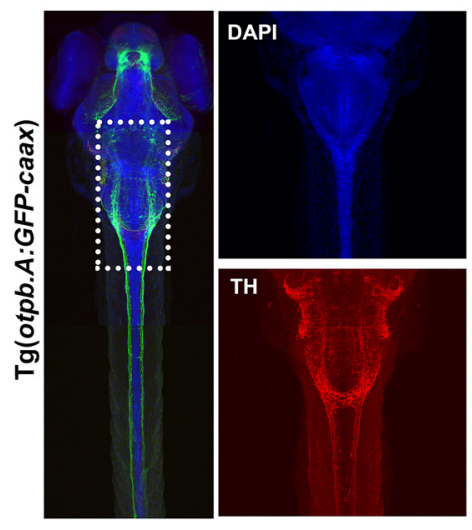

TH

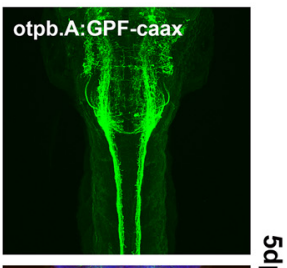

음
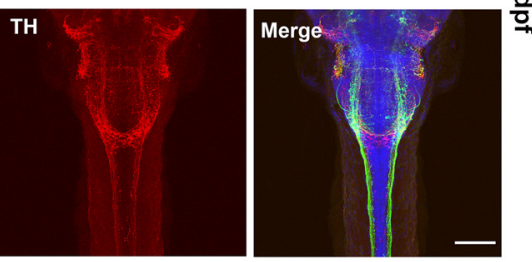

D
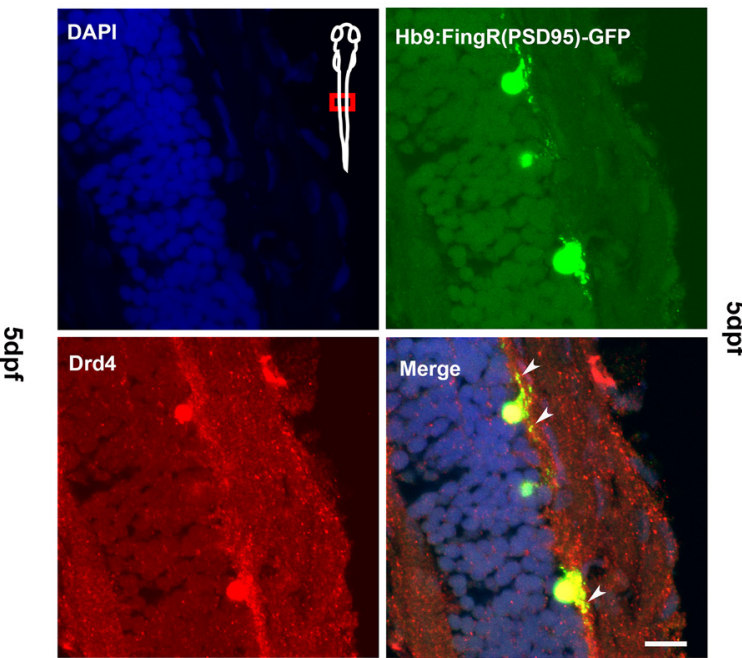

$\stackrel{9}{9}$

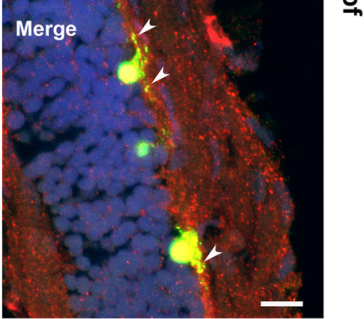

Figure 1. Dopaminergic synapses to spinal cord motor neurons. A, Confocal images, lateral views of zebrafish spinal cord at 5 dpf, dorsal to top; scale bar: $10 \mu \mathrm{m}$. DAPI staining of cell nuclei; anti-Hb9 immunohistochemistry in motor neurons; anti-TH immunohistochemistry of DDT; and double-labeling anti-Hb9 anti-TH shows co-localization (arrows). $\boldsymbol{B}$, Confocal images, dorsal views of zebrafish at $5 \mathrm{dpf}$, rostral top. Left panel shows entire larva with DDT labeled by GFP in Tg(otpb.A:EGFP-caax) line. Higher magnification (scale bar: $50 \mu \mathrm{m}$ ) shows DAPI staining of cell nuclei; GFP labeling of DDT; anti-TH labeling of TH; and co-localization of TH and GFP in DDT. C, Tg(otpb.A:TRFP-caax); Tg(Hb9:FingR(PSD95)-GFP) larvae, confocal images, dorsal views, of spinal cord at 5 dpf (scale bar: $10 \mu \mathrm{m})$. FingR localization of PSD95, expressed in motor neurons, of synapses can be seen neighboring DDT labeled by RFP (arrowheads). D, Anti-D4R labeling co-localizes with FingR GFP expression (scale bar: $10 \mu \mathrm{m})$.

\section{Effects of hypoxia on the development of dopaminergic neurons, DDT, and motor neurons}

To determine where in the CNS hypoxia was exerting its effects, we labeled hypoxic-treated or normoxic-treated 5 dpf zebrafish with anti-TH (Fig. 3C,B). A one-way ANOVA showed there was a significant effect of hypoxia on the number of $\mathrm{TH}^{+}$neurons in the posterior tuberculum (PT; $\left.F_{(3,33)}=13, p<0.01\right)$ with certain hypoxia conditions. The post hoc analysis revealed there was a significantly reduced number of $\mathrm{TH}^{+}$dopaminergic neurons observed with 1- to 2-dpf hypoxia $\left(1 \% \mathrm{pO}_{2}\right)$ and 2- to 3-dpf hypoxia $\left(3 \% \mathrm{pO}_{2}\right)$, but not from 3- to 4-dpf hypoxia $\left(5 \% \mathrm{pO}_{2}\right.$; Fig.
$3 C)$. This was also reflected in whole zebrafish anti-TH quantification by Western blot analysis at $5 \mathrm{dpf}$ following hypoxia, in which larval zebrafish showed significantly decreased TH levels at earlier stages of hypoxia exposure including 1-2 dpf $\left(1 \% \mathrm{pO}_{2}\right)$ and $2-3 \mathrm{dpf}\left(3 \% \mathrm{pO}_{2}\right)$, but not at $3-4 \mathrm{dpf}\left(5 \% \mathrm{pO}_{2}\right)$ compared with normoxic zebrafish $(p=0.91$; Fig. $3 D, E)$. We did not see any errors in pathfinding of the DDT of extraneous midline crossing (Fig. 3B, bottom row); and overall intensity of the DDT descending tracts was unchanged following hypoxia (Fig. 3F).

To determine whether hypoxia could specifically alter CNS circuitry, in the absence of changes in apoptosis or 
A Hypoxia paradigam

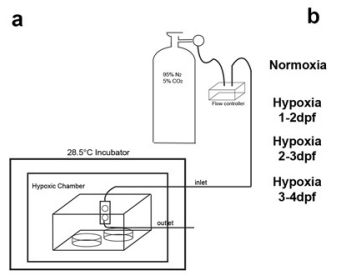

C
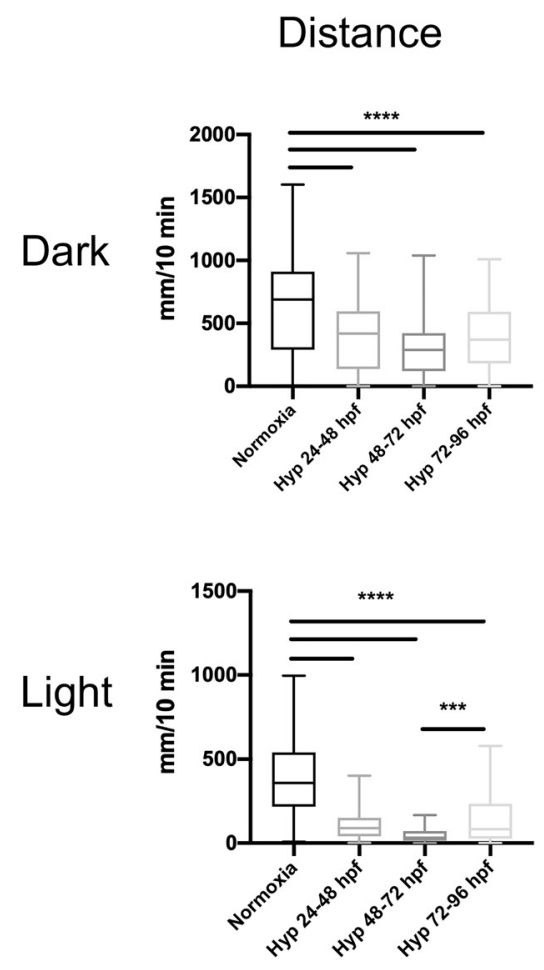

D
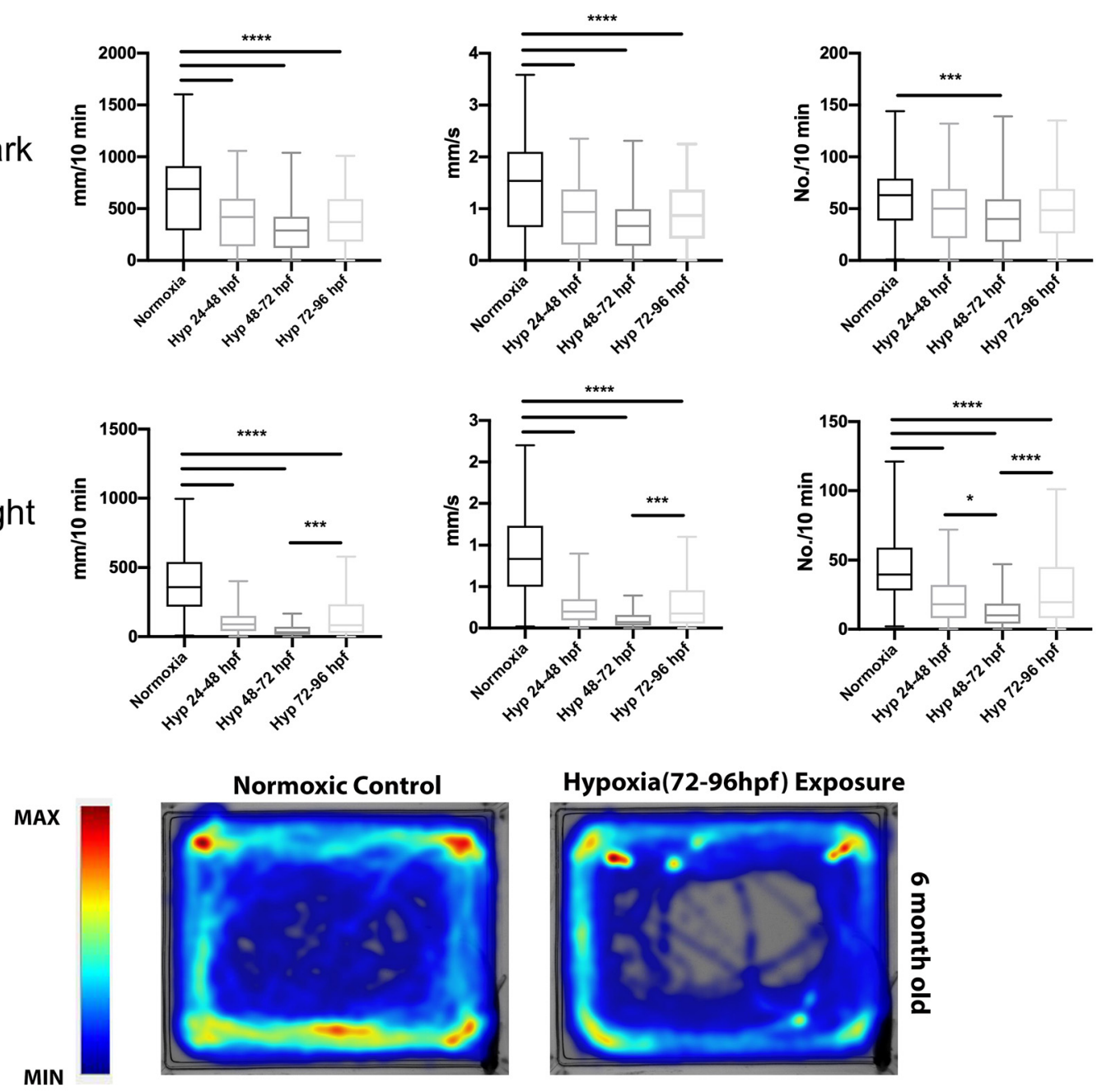

Rotation Frequency

Behavioral paradigm

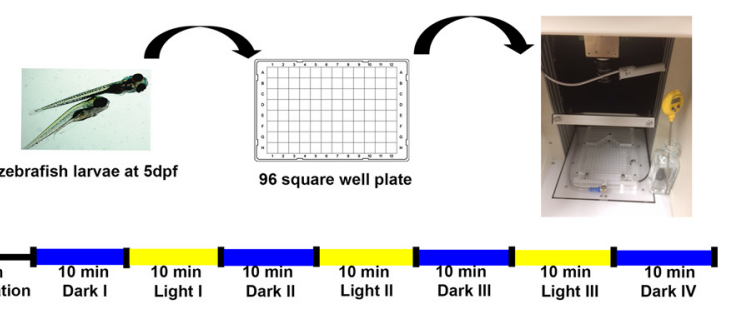

Velocity

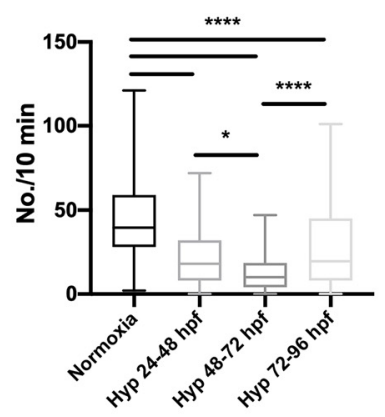

Hypoxia(72-96hpf) Exposure

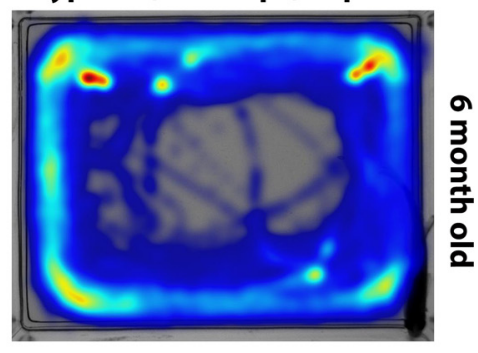

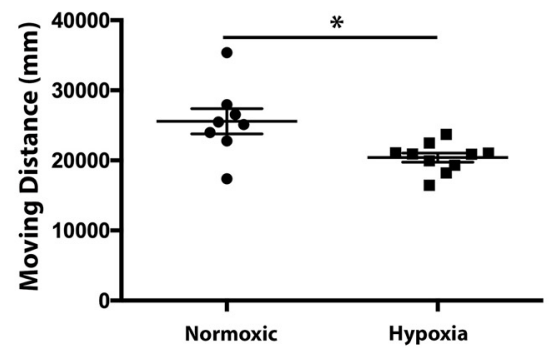
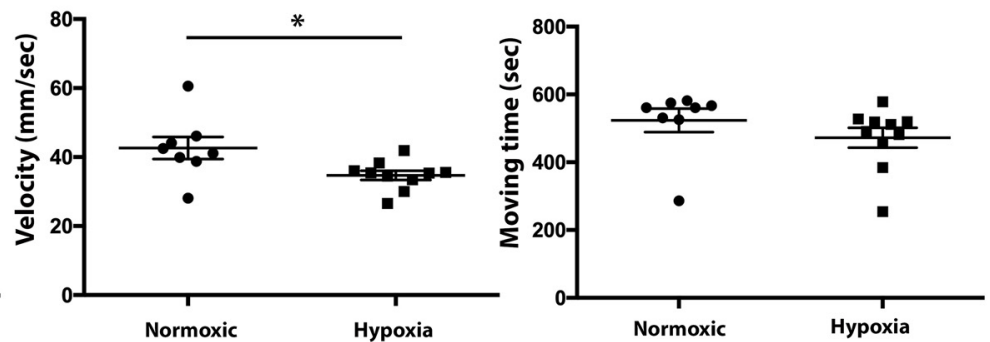
continued

Figure 2. Hypoxia causes impaired motor behavior in larval and adult animals. $\boldsymbol{A}$, Experimental set-up for hypoxia (a) and time line (b) of hypoxia exposure and analysis. $\boldsymbol{B}$, Schematic of behavioral testing paradigm. $\boldsymbol{C}$, Data plots show impaired motor behavior in hypoxia-exposed larvae, each point is a separate animal. Mean shown, ANOVA, $* * * * p<0.0001, * * * p<0.001$. $\boldsymbol{D}$, top, Heat-map of adult zebrafish (six-month-old) exposed to hypoxia (3- to 4-dpf $5 \% \mathrm{pO}_{2}$ ) shows decreased swimming. Below, Hypoxia-exposed zebrafish have persistent decreases in velocity and distance swum as adults animals (normoxia $n=8$, pre-hypoxia $n=10$ ); $* p<0.05$; SD shown).

cell fate that could cause secondary changes (e.g., following hypoxia at 1-2 or 2-3 dpf), we focused analysis of hypoxia at later developmental stages (3-4 dpf with $5 \%$ $\mathrm{pO}_{2}$ ). That is, we did not want to have confounding effects introduced by the more severe defects caused by earlier hypoxia, such as the reduction in $\mathrm{TH}+$ neurons or altered axons (Fig. 3B, top panels). We counted the number of $\mathrm{TH}^{+}$neurons in the PT of the diencephalon at $5 \mathrm{dpf}$ and did not find significant changes in counts following hypoxia exposure (normoxia, $41.9 \pm 2.2$; hypoxia, $34.7 \pm 3.4, t_{(15)}=$ $1.848, p=0.08$; Fig. $3 C$ ). Nor were there any difference in apoptosis (measured by TUNEL+ counts) in hypoxiatreated animals $\left(t_{(12)}=-1.213, p=0.249\right.$; Fig. $\left.3 G-l\right)$. We also evaluated axon pathfinding of the longitudinal DDT track, using the transgenic reporter line Tglotpb.A:egfpcaax) or with anti-TH immunohistochemistry. There were no observed axon pathfinding errors (Fig. $3 B, G$ ), and no change in the intensity of anti-TH labeling [normoxia, 19.09 average intensity units (Al) \pm 2.73 ; hypoxia, $16.5 \pm 1.34 \mathrm{Al}$, $t_{(13)}=0.795, p=0.44$; Fig. $\left.3 E, F\right]$, which is suggestive that the axons are not changing routes. We also characterized the effects of hypoxia on motor neurons in the spinal cord. There were no significant hypoxic effects on proliferation counts $\left(t_{(10)}=0.898, p=0.390\right)$ or apoptosis counts $\left(t_{(12)}=\right.$ $-1.213, p=0.249$ ) in motor neurons (Fig. 3J-M). Together, these data suggest that $5 \% \mathrm{pO}_{2}$ hypoxia from 3 to $4 \mathrm{dpf}$ did not affect dopaminergic or motor neuron cell counts, apoptosis, or proliferation; and did not affect the axonal projections of the DDT.

\section{Effects of hypoxia on the synaptic connectivity of DDT to $\mathrm{MN}$}

We quantified the synapses between the DDT and motor neurons following hypoxia, using quantification of FingR + / $\mathrm{TH}+$ puncta following anti-TH immunohistochemistry; or of FingR+/RFP+ puncta in double transgenic larvae $\mathrm{Tg}$ (otpb.A:TRFP-caax); Tg(Hb9:FingR(PSD95)-GFP). FingR (PSD95)-GFP is an antibody-like protein that label endogenous synaptic PSD-95 with a GFP label (Son et al., 2016). We analyzed the number of motor neurons expressing FingR(PSD95)-GFP (i.e., number of PSD95-GFP+ motor neurons); the co-localized punctate labeling between FingR(PSD95)-GFP and the DDT (i.e., number of putative synapses); and DDT signal intensity (i.e., axonal projections; Fig. $4 A-E)$. In the double transgenic animals, we did not find differences in the number of FingR(PSD95)+ motor neurons $\left(t_{(15)}=1.014, p=0.327\right)$, or in the DDT signal intensity $\left(t_{(15)}=1.219, p=0.229\right.$; Fig. $\left.4 C\right)$. However, we found a significant decreased number of the co-localized punctate number of FingR(PSD95)-GFP onto DDT in hypoxic zebrafish $(120.25 \pm 16.10)$ compared with normoxic zebrafish $\left(372.66 \pm 53.72 ; t_{(15)}=4.267, p<0.01\right.$; Fig. $4 C$ ). Further, when we corrected synapse counts for the number of motor neurons, our results also showed a significant decreased of the number of synapses between the DDT and motor neurons $\left(t_{(15)}=3.955, p<0.05\right)$. We observed similar results for quantification using anti-TH immunohistochemistry in $\operatorname{Tg}\left(H b 9\right.$ :FingR(PSD95)-GFP) larvae $\left(t_{(13)}=2.471\right.$, $p<0.05$; Fig. $4 D, E$ ). Together, these data indicate that there was a significant decrease in DDT to motor neuron synapses following hypoxia.

Similarly, when exposed zebrafish larvae to a dopaminergic D4 receptor antagonist (L-745870) from 72 to 96 hpf, we observed motor impairments in distance, velocity, and rotation frequency [distance $\left(t_{(58)}=3.90, p<0.01\right)$, velocity $\left(t_{(58)}=3.19, p<0.01\right)$, and rotation frequency $\left(t_{(58)}=\right.$ $3.93, p<0.01$ ); Fig. 5; Table 3], similar to the effects of hypoxia exposure. Further, inclusion of the D4 receptor antagonist together with hypoxia exposure did not cause any additional worsening of motor phenotypes, consistent with the two exposures acting via a shared or similar mechanism.

\section{Discussion}

Our work demonstrates that dopaminergic signaling is necessary for the development of the locomotor synaptic circuitry, and that disruption of the synaptic circuitry impairs motor behavior development. Hypoxia disrupts normal co-regulation of locomotion and synaptogenesis, altering CNS circuitry and leading to persistent deficits in motor behavior. These findings refine our understanding of the role for dopamine, which had previously been

Table 1: Spontaneous swimming behavior of larval zebrafish at $5 \mathrm{dpf}$, comparing following hypoxia, to normoxia

\begin{tabular}{|c|c|c|c|c|c|c|}
\hline Treatment & \multicolumn{2}{|c|}{ Swimming distance (mm) } & \multicolumn{2}{|c|}{ Velocity (mm/s) } & \multicolumn{2}{|c|}{$\begin{array}{l}\text { Rotation frequency } \\
\text { (no/10 min) }\end{array}$} \\
\hline Normoxia, $n=30,21 \% \mathrm{pO}_{2}$ & $616.6 \pm 48.3$ & $385.3 \pm 38.3$ & $1.4 \pm 0.1$ & $0.9 \pm 0.1$ & $60.4 \pm 3.2$ & $43.2 \pm 3.4$ \\
\hline Hypoxia $48-72 \mathrm{hpf}, n=25,3 \% \mathrm{pO}_{2}$ & $312.5 \pm 28.8$ & $102.9 \pm 18.8$ & $0.7 \pm 0.1$ & $0.2 \pm 0.1$ & $43.3 \pm 4.4$ & $18.3 \pm 2.6$ \\
\hline Hypoxia $72-96 \mathrm{hpf}, n=30,5 \% \mathrm{pO}_{2}$ & $391.0 \pm 31.6$ & $190.7 \pm 28.7$ & $0.9 \pm 0.1$ & $0.4 \pm 0.1$ & $50.5 \pm 34.1$ & $27.9 \pm 3.3$ \\
\hline
\end{tabular}

Behavioral paradigm used alternating light and dark exposures (see Materials and Methods for full description). 
Table 2: Motor behavior in adult fish that had been exposed to hypoxia as 3- to 4-dpf larvae, compared with normoxic exposure controls

\begin{tabular}{llll}
\hline \multicolumn{1}{c}{ Treatment } & Swimming distance $(\mathrm{mm})$ & Velocity $(\mathrm{mm} / \mathrm{s})$ & Movement duration $(\mathrm{s})$ \\
Control, $n=8$ & $25,575.7 \pm 1793.7$ & $42.6 \pm 3.2$ & $523.4 \pm 34.6$ \\
Pre-hypoxia 72-96 hpf, $n=10,5 \% \mathrm{pO}_{2}$ & $20,285.1 \pm 685.6$ & $34.4 \pm 1.4$ & $454.1 \pm 29.5$ \\
\hline
\end{tabular}

A

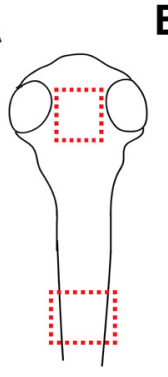

B

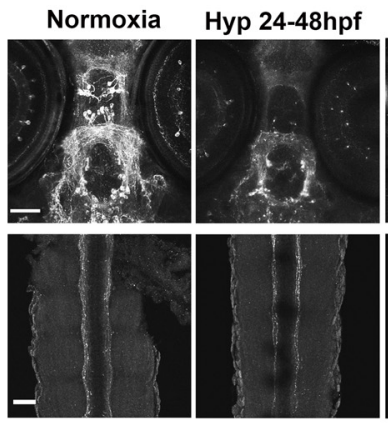

D

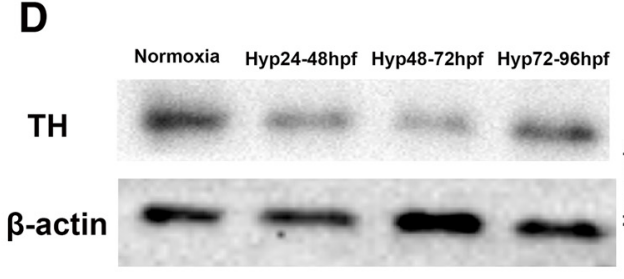

G
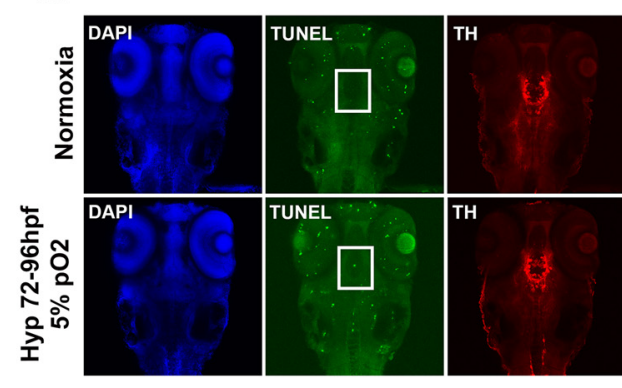
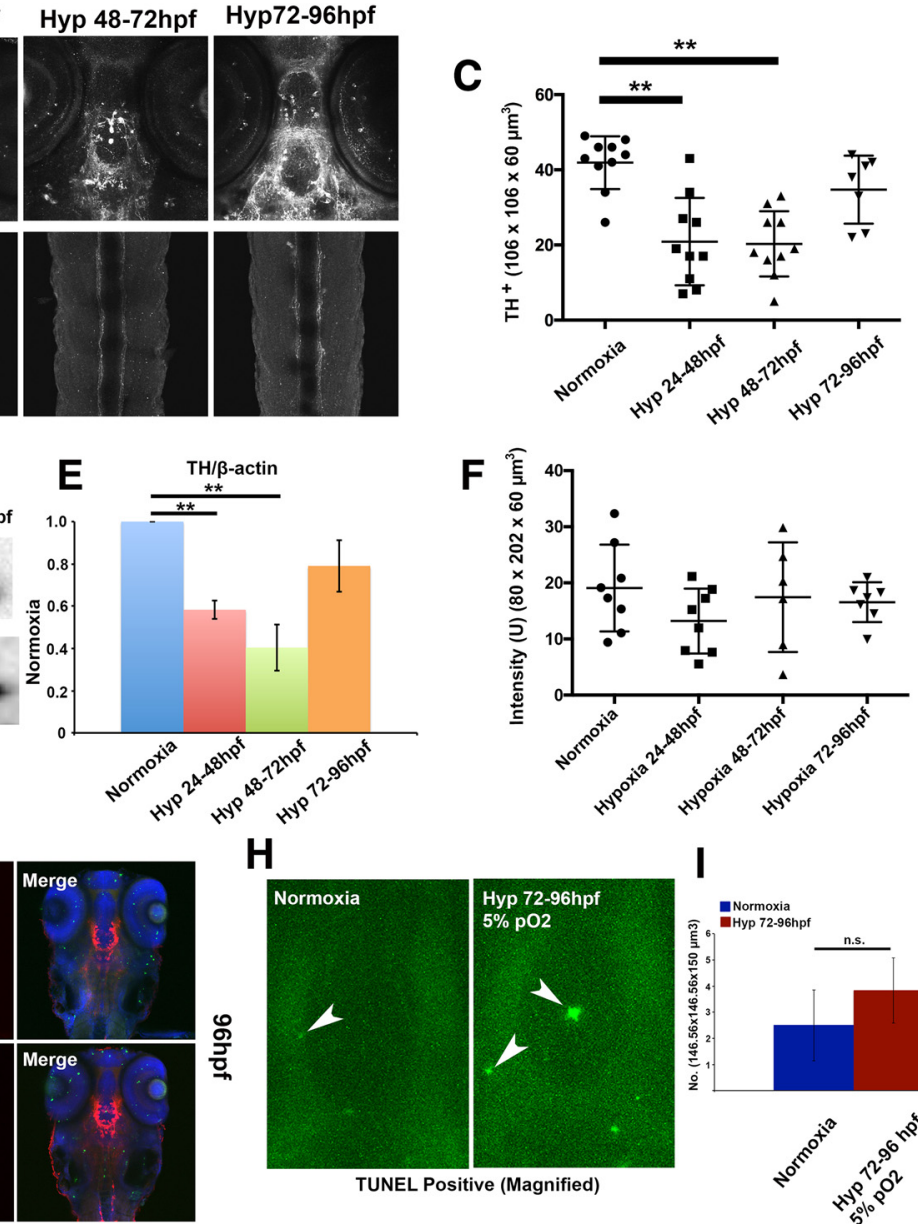

$\mathrm{H}$
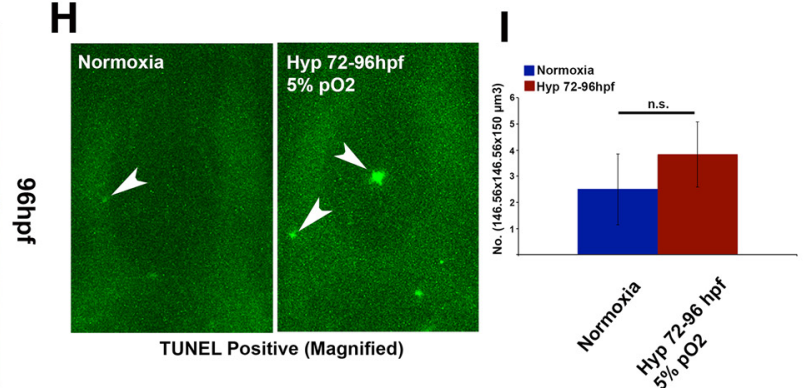

J
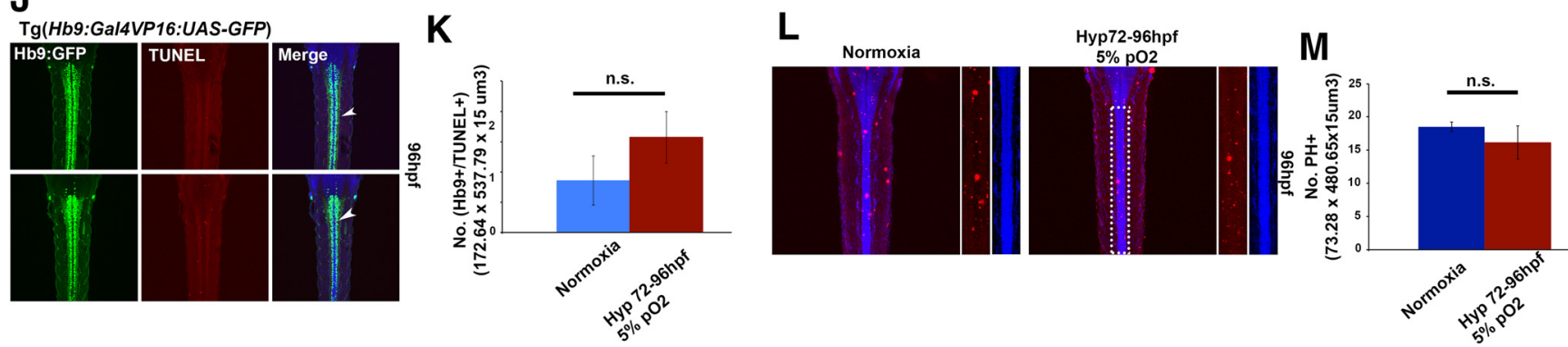

Figure 3. Hypoxia does not alter dopamine or motor neuron numbers, or axon pathfinding of the DDT after hypoxia from 3 to 4 dpf. $\boldsymbol{A}$, Schematic drawing of regions imaged. Top, Diencephalon (for TH+ neurons). Bottom, Spinal cord (for DDT axons). $\boldsymbol{B}$, Confocal images, z-stacks, maximum intensity projections, ventral views, rostral to the top; scale bars: $50 \mu \mathrm{m}$, anti-TH immunohistochemistry. $\boldsymbol{C}$, Quantification of number of TH+ neurons in the diencephalon. $\boldsymbol{D}$, Western blot analysis of whole zebrafish for anti-TH. $\boldsymbol{E}$, AntiTH quantification with SD; $* * p<0.01$. $\boldsymbol{F}$, Intensity of DDT in the spinal cord shows no decrease following hypoxia. $\boldsymbol{G}, \boldsymbol{H}$, No increase in apoptosis posthypoxia in the diencephalon. Confocal images, z-stacks, maximum intensity projections, ventral views, rostral to the top, TUNEL labeling in green. I, Quantification with SD of apoptosis following hypoxia. $\boldsymbol{J}-\boldsymbol{M}$, No increase in apoptosis or proliferation posthypoxia in the spinal cord. Motor neurons identified by co-labeling with $\operatorname{Tg}(H b 9: G F P)$ line. Confocal images, z-stacks, maximum intensity projections, dorsal views, rostral to the top, TUNEL or PH3 (phosphohistone-3) labeling in red. Quantification with SD. n.s., not significant. 


\section{Tg(otpbA:RFP-caax;Hb9:lin/notch:Gal4VP16:zcUAS:PSD95.FingR-GFP)}

A
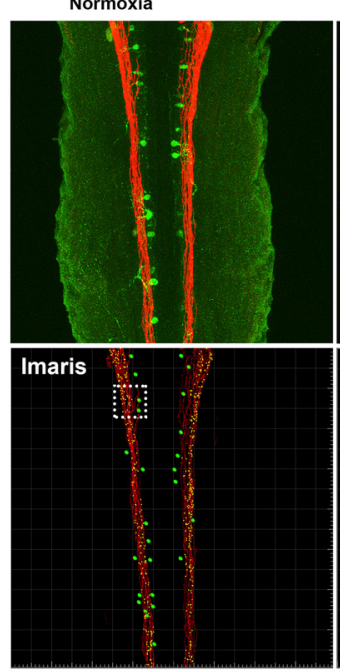

Hyp 72-96hpf

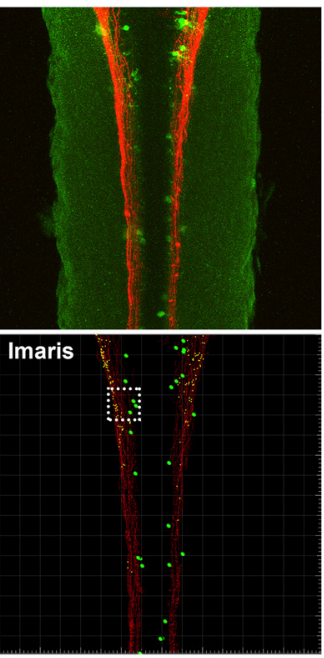

B

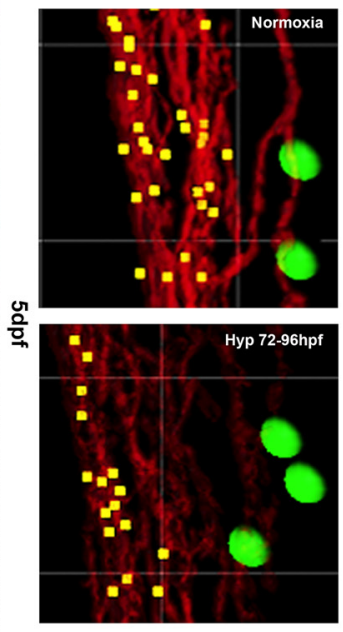

C

Effect of Hypoxia (72-96hpf) on DA-MN synapse

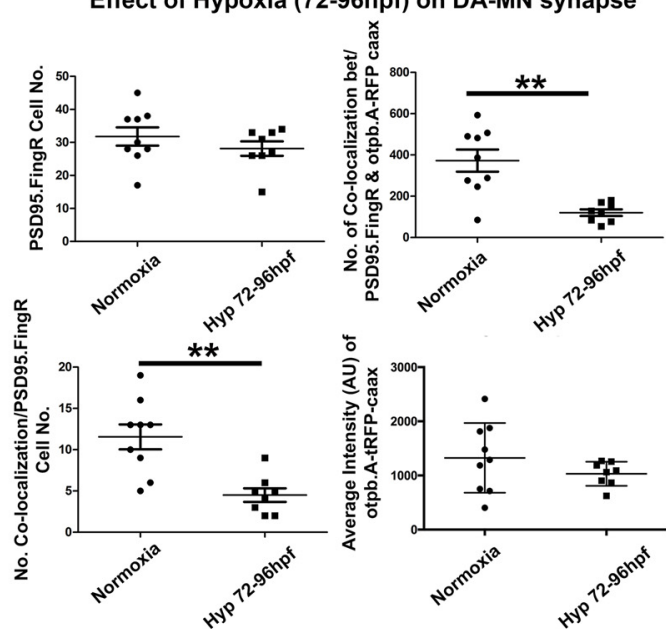

D

\section{Tg(Hb9:lin/notch:gal4vp16;zcUAS:PSD95.FingR-GFP)}
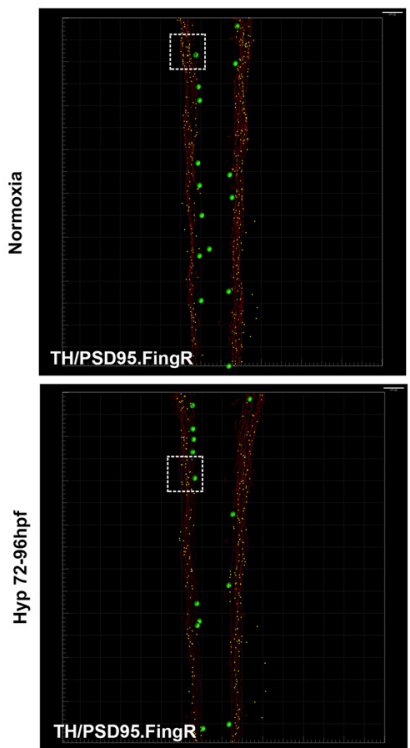
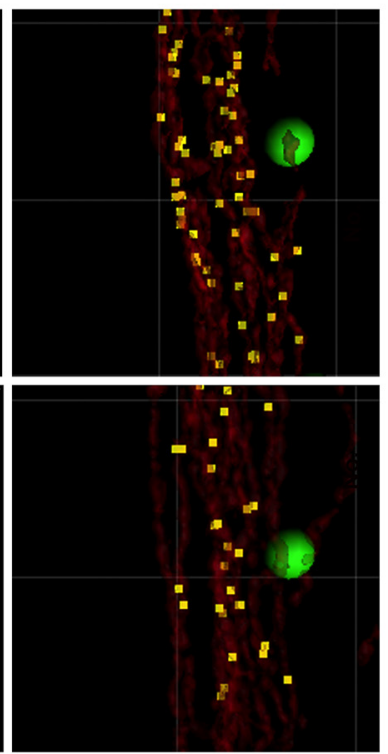
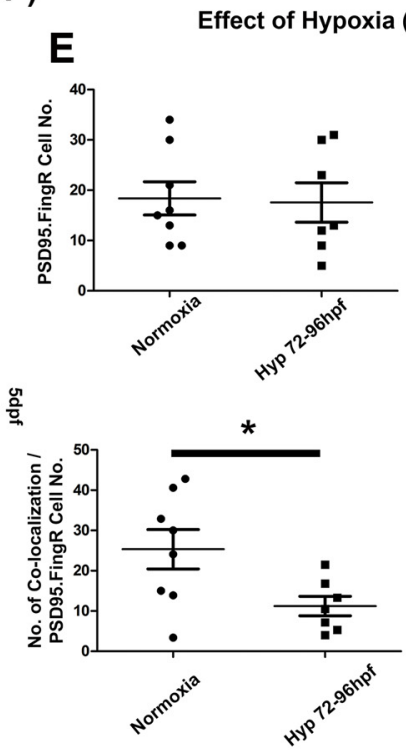
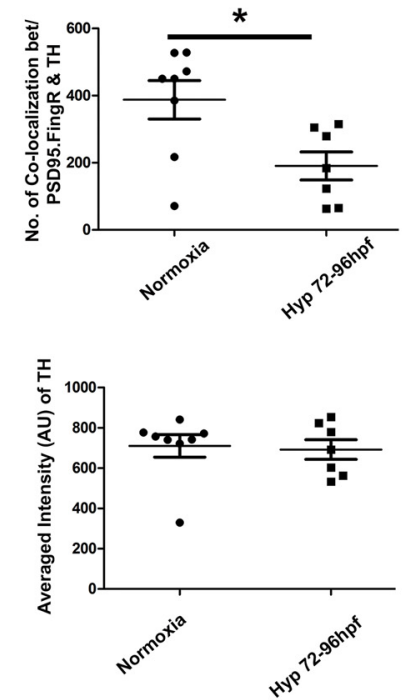

Figure 4. Hypoxia causes a decrease in DDT/motor neuron synapses. $\boldsymbol{A}$, Confocal images of spinal cord of Tg(otpb.A.:TRFP-caax); $\mathrm{Tg}$ (Hb9:FingR(PSD95)-GFP) larvae, anti-GFP, anti-RFP labeling. Rostral to top, DDT labeled in red, motor neurons in green, synapses in yellow. B, Magnified view of IMARIS imaging of synapses. C, Quantification, mean and SD, $* * p<0.01$. Number of motor neurons and intensity of DDT is not affected by hypoxia, but number of synapses is decreased, including when corrected for motor neuron number. $\boldsymbol{D}$, Confocal images of spinal cord of Tg(Hb9:FingR(PSD95)-GFP) larvae with anti-TH anti-GFP immunohistochemistry. Right panels, IMARIS panel image shown to right of corresponding confocal image. E, Quantification with mean and SD shows that number of motor neurons and intensity of DDT is not affected by hypoxia, but number of synapses is decreased, including when corrected for motor neuron number. $* p<0.05$.

demonstrated to be necessary for neurogenesis in the spinal cord, and for proper locomotor development (Popolo et al., 2004; Reimer et al., 2013). Although projections of the A11 group of diencephalic dopaminergic neurons into the spinal cord were first characterized in the 1970s (Björklund and Skagerberg, 1979; Hökfelt et al., 1979), early work on the DDT suggested that the DDT terminated in the dorsal horn and was primarily involved in autonomic functions (Skagerberg et al., 1982). However, TH immunoreactivity was identified near motor neurons in the spinal cord (McLean and Fetcho, 2004b), and functional and behavioral studies revealed the DDT is the source of dopamine involved in motor system development (Lambert et al., 2012), and is necessary for on-going regulation of locomotion behavior (Jay et al., 2015; Sharples et al., 2015). Since we and 

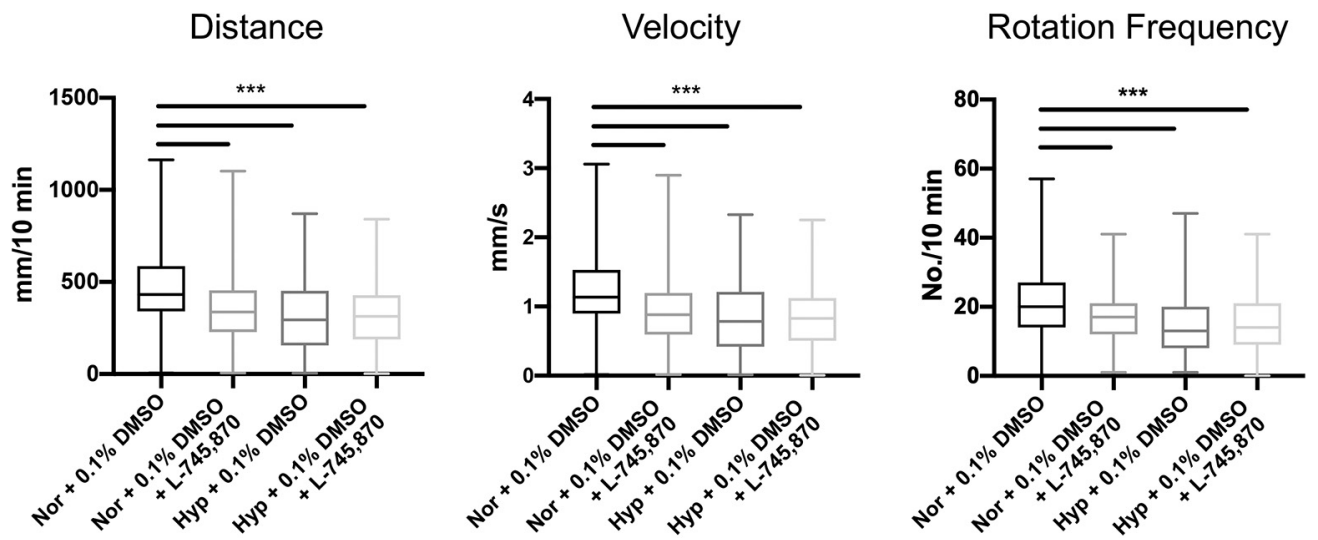

Figure 5. Dopaminergic antagonist exposure impairs zebrafish locomotor development similar to effects of hypoxia. Analysis of zebrafish larvae at $5 \mathrm{dpf}$, following exposure to L-745870 from 72 to $96 \mathrm{hpf}$. Impairments in distance, velocity, and rotation frequency (quantified data in Table 3). Each point is a separate animal. Mean shown, ANOVA; $* * * p<0.001$. Hyp, hypoxia; Nor, normoxia.

others have now shown roles for dopamine in motor circuitry development and in motor behavior development, this raises the possibility for a coordinating role for dopamine signaling in these processes.

We found new evidence regarding the role of dopamine in co-regulation of circuitry development with locomotor development. We used the zebrafish model system, characterizing the motor neuron synapses of the spinal cord and the descending dopamine projections to them. We found a decrease in DDT to motor neuron synapses, which was associated with a decrease in swimming behaviors compared with normoxic conditions. Previous work in vertebrates as well as in invertebrates has shown that developmental hypoxia causes axonal pathfinding errors in telencephalic neurons, through activation of the hypoxia-inducible factor (hif1) pathway (Stevenson et al., 2012; Xing et al., 2015). Hypoxia has also been noted to affect synapse development (Curristin et al., 2002; Segura et al., 2016; Zhuravin et al., 2019), but in this work we identified that hypoxia could act on dopaminergic signaling to affect synapse development, including effects on synaptic circuitry, and short- and long-term behavioral effects. However, we cannot exclude that hypoxia might have multifactorial impacts on behavior and locomotor development, for example, that hypoxia could be affecting myelin development or function; or of other aspects of circuitry, for example, motor neuron to muscle synapses.

This work also found new evidence for co-localization of DDT descending tract synapses with motor neuron synapses. This finding, if also observed in humans, could provide novel insights into unexplored aspects of human locomotion as well as into pathologies associated with premature birth. In mammals the A11 DDT is also dopaminergic (Koblinger et al., 2014). Premature birth is associated with hypoxic exposures during a critical period of brain development (Martin et al., 2011b, 2012) from 24 to 36 weeks PCA (postconception age), and includes when axon and synaptic connections are forming. This period includes corticospinal tract extension and innervation of spinal primary motor neurons; development of the corpus callosum; thalamocortical axon formation; and the connection of cortical with subcortical areas (Eyre et al., 2000; ten Donkelaar et al., 2004; Haynes et al., 2005; Ren et al., 2006; Kostovic and Jovanov-Milosevic, 2006; Vasung et al., 2010). Hypoxia from prematurity is strongly correlated with the risk for neuromotor impairment (Martin et al., 2011 b; Salmaso et al., 2014) and can affect up to $60 \%$ of infants (Williams et al., 2010). The hypoxia is secondary to a complex set of conditions, including utero-placental insufficiency and immaturity of the cardiopulmonary system. Following birth, premature infants can experience up to 600 hypoxic episodes per week, each lasting at least $>10$ s (Martin et al., 2011b).

The impaired locomotion from the developmental hypoxia persists into adulthood. The findings may offer insight into the adverse motor effects of prematurity and accompanying hypoxia. Each year, 13 million infants worldwide are born prematurely (Beck et al., 2010; Martin et al., 2011a), and motor impairment affects a majority of children born prematurely (Williams et al., 2010) with little insight into pathophysiology and no available treatments. Key future research areas include understanding the interaction between myelination and oligodendrocytes, the effects on circuitry from hypoxia, and the relationship of

Table 3: Motor behavior in zebrafish larvae following hypoxia or normoxia, with or without exposure to a dopaminergic D4 receptor antagonist (L-745870) from 72 to $96 \mathrm{hpf}$

\begin{tabular}{llll}
\hline \multicolumn{1}{c}{ Treatment } & Swimming distance $(\mathrm{mm})$ & Velocity $(\mathrm{mm} / \mathrm{s})$ & Rotation frequency (no/10 min) \\
Normoxia, $n=24,21 \% \mathrm{pO}_{2}$ & $655.4 \pm 71.3$ & $1.7 \pm 0.2$ & $54.0 \pm 4.4$ \\
Hypoxia 72-96 hpf, $n=24,5 \% \mathrm{pO}_{2}$ & $361.7 \pm 54.1$ & $0.9 \pm 0.1$ & $41.5 \pm 5.3$ \\
D4 control (0.1\% DMSO), $n=12$ & $581.7 \pm 113.5$ & $1.3 \pm 0.3$ & $52.1 \pm 8.8$ \\
D4 antagonist (L-745870), $n=12$ & $282.5 \pm 37.4$ & $0.6 \pm 0.1$ & $23.4 \pm 3.7$
\end{tabular}


hypoxia-related circuitry changes to complex neurobehavioral disorders such as autism (Yuen et al., 2014; Modabbernia et al., 2017; Wang et al., 2018; Yang et al., 2018; Chen et al., 2019).

\section{References}

Back SA (2014) Cerebral white and gray matter injury in newborns: New insights into pathophysiology and management. Clin Perinatol 41:1-24.

Barrett RD, Bennet L, Davidson J, Dean JM, George S, Emerald BS, Gunn AJ (2007) Destruction and reconstruction: Hypoxia and the developing brain. Birth Defects Res C Embryo Today 81:163-176.

Bass JL, Corwin M, Gozal D, Moore C, Nishida H, Parker S, Schonwald A, Wilker RE, Stehle S, Kinane TB (2004) The effect of chronic or intermittent hypoxia on cognition in childhood: A review of the evidence. Pediatrics 114:805-816.

Beck S, Wojdyla D, Say L, Betran AP, Merialdi M, Requejo JH, Rubens C, Menon R, Van Look PF (2010) The worldwide incidence of preterm birth: A systematic review of maternal mortality and morbidity. Bull World Health Organ 88:31-38.

Berg EM, Bjornfors ER, Pallucchi I, Picton LD, Manira AE (2018) Principles governing locomotion in vertebrates: Lessons from zebrafish. Front Neural Circuits 12:73.

Björklund A, Skagerberg G (1979) Evidence for a major spinal cord projection from the diencephalic A11 dopamine cell group in the rat using transmitter-specific fluorescent retrograde tracing. Brain Res 177:170-175.

Bonkowsky JL, Son JH (2018) Hypoxia and connectivity in the developing vertebrate nervous system. Dis Model Mech 11.

Bonkowsky JL, Wang X, Fujimoto E, Lee JE, Chien CB, Dorsky RI (2008) Domain-specific regulation of foxP2 CNS expression by lef1. BMC Dev Biol 8:103

Buss RR, Drapeau P (2001) Synaptic drive to motoneurons during fictive swimming in the developing zebrafish. J Neurophysiol 86:197-210.

Chang AJ, Bargmann Cl (2008) Hypoxia and the HIF-1 transcriptional pathway reorganize a neuronal circuit for oxygen-dependent behavior in Caenorhabditis elegans. Proc Natl Acad Sci USA 105:7321-7326.

Chen PY, Tsai YW, Cheng YJ, Giangrande A, Chien CT (2019) Glial response to hypoxia in mutants of NPAS1/3 homolog Trachealess through $\mathrm{Wg}$ signaling to modulate synaptic bouton organization. PLoS Genet 15:e1007980.

Curristin SM, Cao A, Stewart WB, Zhang H, Madri JA, Morrow JS, Ment LR (2002) Disrupted synaptic development in the hypoxic newborn brain. Proc Natl Acad Sci USA 99:15729-15734.

D'Elia KP, Dasen JS (2018) Development, functional organization, and evolution of vertebrate axial motor circuits. Neural Dev 13:10.

Di Fiore JM, Poets CF, Gauda E, Martin RJ, MacFarlane P (2016) Cardiorespiratory events in preterm infants: Etiology and monitoring technologies. J Perinatol 36:165-171.

Eyre JA, Miller S, Clowry GJ, Conway EA, Watts C (2000) Functional corticospinal projections are established prenatally in the human foetus permitting involvement in the development of spinal motor centres. Brain 123 (Pt 1):51-64.

Fanaroff AA, Stoll BJ, Wright LL, Carlo WA, Ehrenkranz RA, Stark AR, Bauer CR, Donovan EF, Korones SB, Laptook AR, Lemons JA, Oh W, Papile LA, Shankaran S, Stevenson DK, Tyson JE, Poole WK; NICHD Neonatal Research Network (2007) Trends in neonatal morbidity and mortality for very low birthweight infants. Am J Obstet Gynecol 196:147.e1-8.

Fujimoto E, Stevenson TJ, Chien CB, Bonkowsky JL (2011) Identification of a dopaminergic enhancer indicates complexity in vertebrate dopamine neuron phenotype specification. Dev Biol 352:393-404.

Gao J, Stevenson TJ, Douglass AD, Barrios JP, Bonkowsky JL (2018) The midline axon crossing decision is regulated through an activity-dependent mechanism by the NMDA receptor. eNeuro 5 . ENEURO.0389-17.2018.

Garcia-Campmany L, Stam FJ, Goulding M (2010) From circuits to behaviour: Motor networks in vertebrates. Curr Opin Neurobiol 20:116-125.

Gozzo Y, Vohr B, Lacadie C, Hampson M, Katz KH, MallerKesselman J, Schneider KC, Peterson BS, Rajeevan N, Makuch RW, Constable RT, Ment LR (2009) Alterations in neural connectivity in preterm children at school age. Neuroimage 48:458-463.

Gross GG, Junge JA, Mora RJ, Kwon HB, Olson CA, Takahashi TT, Liman ER, Ellis-Davies GC, McGee AW, Sabatini BL, Roberts RW, Arnold DB (2013) Recombinant probes for visualizing endogenous synaptic proteins in living neurons. Neuron 78:971-985.

Gutnick A, Blechman J, Kaslin J, Herwig L, Belting HG, Affolter M, Bonkowsky JL, Levkowitz G (2011) The hypothalamic neuropeptide oxytocin is required for formation of the neurovascular interface of the pituitary. Dev Cell 21:642-654.

Haynes RL, Borenstein NS, Desilva TM, Folkerth RD, Liu LG, Volpe JJ, Kinney HC (2005) Axonal development in the cerebral white matter of the human fetus and infant. J Comp Neurol 484:156167.

Hintz SR, Kendrick DE, Wilson-Costello DE, Das A, Bell EF, Vohr BR, Higgins RD; NICHD Neonatal Research Network (2011) Earlychildhood neurodevelopmental outcomes are not improving for infants born at $<25$ weeks' gestational age. Pediatrics 127:62-70.

Hökfelt T, Phillipson O, Goldstein M (1979) Evidence for a dopaminergic pathway in the rat descending from the A11 cell group to the spinal cord. Acta Physiol Scand 107:393-395.

Jay M, De Faveri F, McDearmid JR (2015) Firing dynamics and modulatory actions of supraspinal dopaminergic neurons during zebrafish locomotor behavior. Curr Biol 25:435-444.

Koblinger K, Füzesi T, Ejdrygiewicz J, Krajacic A, Bains JS, Whelan PJ (2014) Characterization of A11 neurons projecting to the spinal cord of mice. PLoS One 9:e109636.

Kostovic I, Jovanov-Milosevic N (2006) The development of cerebral connections during the first $20-45$ weeks' gestation. Semin Fetal Neonatal Med 11:415-422.

Kwan KM, Fujimoto E, Grabher C, Mangum BD, Hardy ME, Campbell DS, Parant JM, Yost HJ, Kanki JP, Chien CB (2007) The Tol2kit: A multisite gateway-based construction kit for Tol2 transposon transgenesis constructs. Dev Dyn 236:3088-3099.

Lambert AM, Bonkowsky JL, Masino MA (2012) The conserved dopaminergic diencephalospinal tract mediates vertebrate locomotor development in zebrafish larvae. J Neurosci 32:13488-13500.

Martin JA, Hamilton BE, Ventura SJ, Osterman MJ, Kirmeyer S, Mathews TJ, Wilson EC (2011) Births: Final data for 2009. Natl Vital Stat Rep 60:1-70.

Martin RJ, Wang K, Köroğlu O, Di Fiore J, Kc P (2011) Intermittent hypoxic episodes in preterm infants: Do they matter? Neonatology 100:303-310.

Martin RJ, Di Fiore JM, Macfarlane PM, Wilson CG (2012) Physiologic basis for intermittent hypoxic episodes in preterm infants. Adv Exp Med Biol 758:351-358.

Mathews TJ, Miniño AM, Osterman MJ, Strobino DM, Guyer B (2011) Annual summary of vital statistics: 2008. Pediatrics 127:146-157.

McLean DL, Fetcho JR (2004a) Ontogeny and innervation patterns of dopaminergic, noradrenergic, and serotonergic neurons in larval zebrafish. J Comp Neurol 480:38-56.

McLean DL, Fetcho JR (2004b) Relationship of tyrosine hydroxylase and serotonin immunoreactivity to sensorimotor circuitry in larval zebrafish. J Comp Neurol 480:57-71.

Milash B, Gao J, Stevenson TJ, Son JH, Dahl T, Bonkowsky JL (2016) Temporal dysynchrony in brain connectivity gene expression following hypoxia. BMC Genomics 17:334.

Modabbernia A, Velthorst E, Reichenberg A (2017) Environmental risk factors for autism: An evidence-based review of systematic reviews and meta-analyses. Mol Autism 8:13.

Mullen KM, Vohr BR, Katz KH, Schneider KC, Lacadie C, Hampson M, Makuch RW, Reiss AL, Constable RT, Ment LR (2011) Preterm 
birth results in alterations in neural connectivity at age 16 years. Neuroimage 54:2563-2570.

Pocock R, Hobert O (2010) Hypoxia activates a latent circuit for processing gustatory information in C. elegans. Nat Neurosci 13:610-614.

Popolo M, McCarthy DM, Bhide PG (2004) Influence of dopamine on precursor cell proliferation and differentiation in the embryonic mouse telencephalon. Dev Neurosci 26:229-244.

Reimer MM, Norris A, Ohnmacht J, Patani R, Zhong Z, Dias TB, Kuscha V, Scott AL, Chen YC, Rozov S, Frazer SL, Wyatt C, Higashijima S, Patton EE, Panula P, Chandran S, Becker T, Becker CG (2013) Dopamine from the brain promotes spinal motor neuron generation during development and adult regeneration. Dev Cell 25:478-491.

Ren T, Anderson A, Shen WB, Huang H, Plachez C, Zhang J, Mori S, Kinsman SL, Richards LJ (2006) Imaging, anatomical, and molecular analysis of callosal formation in the developing human fetal brain. Anat Rec A Discov Mol Cell Evol Biol 288:191-204.

Saigal S, Doyle LW (2008) An overview of mortality and sequelae of preterm birth from infancy to adulthood. Lancet 371:261-269.

Salmaso N, Tomasi S, Vaccarino FM (2014) Neurogenesis and maturation in neonatal brain injury. Clin Perinatol 41:229-239.

Segura I, Lange C, Knevels E, Moskalyuk A, Pulizzi R, Eelen G, Chaze T, Tudor C, Boulegue C, Holt M, Daelemans D, Matondo M, Ghesquière B, Giugliano M, Ruiz de Almodovar $C$, Dewerchin $M$, Carmeliet P (2016) The oxygen sensor PHD2 controls dendritic spines and synapses via modification of filamin A. Cell Rep 14:2653-2667.

Sharples SA, Humphreys JM, Jensen AM, Dhoopar S, Delaloye N, Clemens S, Whelan PJ (2015) Dopaminergic modulation of locomotor network activity in the neonatal mouse spinal cord. J Neurophysiol 113:2500-2510.

Skagerberg G, Björklund A, Lindvall O, Schmidt RH (1982) Origin and termination of the diencephalo-spinal dopamine system in the rat. Brain Res Bull 9:237-244.

Son JH, Keefe MD, Stevenson TJ, Barrios JP, Anjewierden S, Newton JB, Douglass AD, Bonkowsky JL (2016) Transgenic FingRs for live mapping of synaptic dynamics in genetically-defined neurons. Sci Rep 6:18734.
Stevenson TJ, Trinh T, Kogelschatz C, Fujimoto E, Lush ME, Piotrowski T, Brimley CJ, Bonkowsky JL (2012) Hypoxia disruption of vertebrate CNS pathfinding through ephrinB2 Is rescued by magnesium. PLoS Genet 8:e1002638.

ten Donkelaar HJ, Lammens M, Wesseling P, Hori A, Keyser A, Rotteveel J (2004) Development and malformations of the human pyramidal tract. J Neurol 251:1429-1442.

Valdez SR, Patterson SI, Ezquer ME, Torrecilla M, Lama MC, Seltzer AM (2007) Acute sublethal global hypoxia induces transient increase of GAP-43 immunoreactivity in the striatum of neonatal rats. Synapse 61:124-137.

Vasung L, Huang $H$, Jovanov-Milošević $N$, Pletikos $M$, Mori $S$, Kostović I (2010) Development of axonal pathways in the human fetal fronto-limbic brain: Histochemical characterization and diffusion tensor imaging. J Anat 217:400-417.

Wang F, Yang YJ, Yang N, Chen XJ, Huang NX, Zhang J, Wu Y, Liu Z, Gao X, Li T, Pan GQ, Liu SB, Li HL, Fancy SPJ, Xiao L, Chan JR, Mei $F$ (2018) Enhancing oligodendrocyte myelination rescues synaptic loss and improves functional recovery after chronic hypoxia. Neuron 99:689-701 e5.

Williams J, Lee KJ, Anderson PJ (2010) Prevalence of motor-skill impairment in preterm children who do not develop cerebral palsy: A systematic review. Dev Med Child Neurol 52:232-237.

Xing L, Son JH, Stevenson TJ, Lillesaar C, Bally-Cuif L, Dahl T, Bonkowsky JL (2015) A serotonin circuit acts as an environmental sensor to mediate midline axon crossing through EphrinB2. J Neurosci 35:14794-14808.

Yang LQ, Chen M, Zhang JL, Ren DL, Hu B (2018) Hypoxia delays oligodendrocyte progenitor cell migration and myelin formation by suppressing Bmp2b signaling in larval zebrafish. Front Cell Neurosci 12:348

Yuen TJ, Silbereis JC, Griveau A, Chang SM, Daneman R, Fancy SPJ, Zahed H, Maltepe E, Rowitch DH (2014) Oligodendrocyte-encoded HIF function couples postnatal myelination and white matter angiogenesis. Cell 158:383-396.

Zhuravin IANM, Dubrovskaya DS, Vasilev TY, Postnikova AV Zaitsev (2019) Prenatal hypoxia produces memory deficits associated with impairment of long-term synaptic plasticity in young rats. Neurobiol Learn Mem 164:107066. 